\title{
Reclutamiento y selección virtual por competencias
}

\author{
Recruitment and virtual selection by skills
}

\section{RESUMEN}

Las Empresas globalizadas y nacionales que se desarrollan en un mundo de turbulencia e incertidumbre como consecuencia de la pandemia COVID-19, que ha generado inestabilidad política, crisis económica, pérdida de puestos de trabajo, entre otros aspectos restrictivos, requieren de una recuperación sostenible, inclusiva y resiliente mediante la construcción de plataformas tecnológicas digitales para optimizar la gestión empresarial. En ese orden de ideas, las empresas requieren reclutar, seleccionar y contratar personal para sus diferentes posiciones de trabajo, dotados de conocimientos de ciencia, tecnología y humanidades que las sociedades del conocimiento así lo demandan y de las habilidades blandas como la inteligencia emocional requeridas para asegurar un desarrollo eficiente, eficaz y efectivo en el cumplimiento de las funciones o tareas asignadas. La pandemia que es letal en el campo de salud física y mental de las personas, nos ha permitido utilizar herramientas digitales como es el trabajo y la educación remota o virtual con resultados satisfactorios utilizando las redes sociales para garantizar el desarrollo de las economías de los países. Las empresas aplican el reclutamiento, selección y contratación del personal por competencias en forma virtual a través del desarrollo de sus procesos técnicos, minimizando costos y maximizando resultados.

Palabras Clave: Reclutamiento; selección; contratación; virtual; competencias.

\begin{abstract}
Globalized and national companies that develop in a world of turbulence and uncertainty as a result of the COVID-19 pandemic, which has generated political instability, economic crisis, loss of jobs, among other restrictive aspects, require a sustainable, inclusive and resilient by building digital technology platforms to optimize business management. In that order of ideas, companies need to recruit, select and hire personnel for their different work positions, endowed with knowledge of science, technology and humanities that knowledge societies demand and with soft skills such as emotional intelligence required to ensure efficient, effective and effective development in the fulfillment of the assigned functions or tasks. The
\end{abstract}

\section{Luis Fernando Valeriano} Ortiz

lvalerianoo@unmsm.edu.pe ORCID: https://orcid.org/00000001-5288-5726

Universidad Nacional Mayor de San Marcos. Lima, Perú

Presentado: 13/07/2021 - Aceptado: 31/08/2021 - Publicado: 23/12/2021

(C) Los autores. Este artículo es publicado por la revista Gestión en el Tercer Milenio de la Facultad de Ciencias Administrativas Universidad Nacional Mayor de San Marcos. Este es un artículo de acceso abierto, distribuido bajo los términos de la licencia Creative Commons Atribución 4.0 Internacional (CC BY 4.0) [https://creativecommons.org/licenses/by/4.0/deed.es] que permite el uso, distribución y reproducción en cualquier medio, siempre que la obra original sea debidamente citada de su fuente original. 
pandemic, which is lethal in the field of physical and mental health of people, has allowed us to use digital tools such as work and remote or virtual education with satisfactory results using social networks to guarantee the development of the economies of the countries. Companies apply the recruitment, selection and hiring of personnel by competencies in a virtual way through the development of their technical processes, minimizing costs and maximizing results.

Keywords: Recruitment; selection; hiring; virtual; competencies.

\section{INTRODUCCIÓN}

En esta era de la globalización de los mercados, de la transformación digital, de la competitividad, de la gestión del cambio, de generar una cultura e identidad empresarial, de la calidad de los productos y/servicios, de la conectividad de generar vínculos con los clientes, usuarios o consumidores, de la innovación y desarrollo, y fundamentalmente de la revalorización del talento humano como el intangible más importante de la gestión empresarial, se hace necesario que las empresas apuesten por la meritocracia en sus procesos de reclutamiento y selección de personal para garantizar la oferta y demanda laboral con transparencia y posibilitar oportunidades laborales basadas en las competencias que el mercado exige.

\section{OBJETO DEL ARTÍCULO}

Desarrollar el marco teórico y práctico de los procesos de reclutamiento, selección y contratación de personal en forma virtual o remota en el ámbito empresarial.

\section{ARGUMENTACIÓN}

Las empresas en las últimas décadas utilizan plataformas tecnológicas virtuales para sus procesos de reclutamiento, selección y contratación de su personal, generándoles mayores ventajas competitivas para atraer, desarrollar y retener personal calificado de acuerdo al perfil del puesto de trabajo deseado, para tal efecto se toman en cuenta los siguientes procesos técnicos:

\section{Marketing Personal}

Es la estrategia personal de la imagen de una persona, para fines de su proyección personal y laboral, en el ámbito en que se desarrolla.

Es la percepción de las personas a partir de sus valores (patrones de conducta), ética (moral), sus conocimientos, habilidades blandas, apariencia física, etc.

\section{Objetivo del Marketing Personal}

Mostrar los mejores patrones de conducta, valores, mayores capacidades, fortalezas y oportunidades que tiene una persona, para potenciar su marca personal, con el objeto de posicionarse en el mercado laboral como persona proactiva y capaz de alcanzar objetivos y metas en forma eficiente y eficaz.

\section{¿Cómo se logra el marketing personal?}

- Participando en eventos relacionados con tu profesión.

- Mantener tu página de LinkedIn siempre actualizada.

- Tener visión de apostar por el futuro.

- Reconocer tus competencias laborales.

- Tener proyecciones de emprendimiento.

- Apostar por una capacitación y especialización permanente

- Teniendo deontología laboral y profesional.

\section{Cuáles son las 4P's del "marketing personal"}

- Producto: Poner atención a la marca personal.

- Plaza: Reconocer los escenarios donde se puede ser exitoso.

- Precio: Identificar tu formación y experiencia laboral en relación a tu valor económico en el mercado.

- Promoción: HV, entrevista laboral y tu "marca digital". 


\section{Reclutamiento de Personal Virtual}

Comprende las siguientes fases:

\section{Perfil del Puesto de Trabajo}

Se elabora el perfil del puesto de trabajo teniendo en cuenta la siguiente estructura:

1. Nomenclatura del cargo: nombre del puesto

2. Naturaleza del cargo: función resumen del puesto

3. Funciones específicas del cargo: se describen las funciones

4. Requerimientos mínimos

4.1. Formación académica: básica regular y universitaria

4.2. Experiencia laboral/profesional: anteriores empresas

\section{Presupuesto de Personal}

El puesto debe estar previsto en el presupuesto de personal.

1. Partida presupuestal o centro de costo del puesto.

2. Estructura remunerativa del puesto.

3. En el sector público esté previsto en el CAP.

\section{Definiciones de Reclutamiento de Personal}

- Para la Real Academia Española (RAE, s.f.) es la "acción y efecto de reclutar. Reunir gente para un propósito determinado".

- Dolan et al. (2007) en su libro La Gestión de los Recursos Humanos menciona que "es un conjunto de actividades y procesos que se realizan para conseguir un número suficiente de personas calificadas, de forma que la organización pueda seleccionar a aquellas más adecuadas para cubrir sus necesidades de trabajo" (p. 109).

- Gómez-Mejía et al. (2008) en su libro Gestión de Recursos Humanos señala que "es el proceso por el que se genera un conjunto de candidatos calificados para un determinado puesto de trabajo es el primer paso del proceso de contratación de personal" (p. 190).

\section{Reclutamiento de Personal Virtual}

Las empresas acuden a dos fuentes: internas y externas para el reclutamiento de personal en el ámbito empresarial

\section{Fuentes Internas.}

Se produce cuando surge la necesidad de cubrir un puesto de trabajo y para ello la empresa acude a la promoción de sus colaboradores (movimientos verticales) o a traslados de éstos (movimientos horizontales) utilizando el intranet empresarial.

\section{Aspectos positivos.}

- Nivel de conocimiento del candidato sobre la organización y viceversa. Menor margen de error.

- Los trabajadores se sienten más seguros y asocian sus intereses a largo plazo con los de la organización.

- La posibilidad de promocionarse internamente aumenta la motivación. Se aprovechan las inversiones en formación realizadas en el empleado.

- Desarrolla un cierto grado de competencia interna.

- Menor costo que el reclutamiento externo, ya que no se necesitan gastos de publicidad u otros medios.

\section{Aspectos negativos.}

- Dificultad de encontrar internamente en la organización, a la persona con las calificaciones requeridas por el puesto.

- Puede dar lugar a conflictos internos, generándose desmotivación y falta de cooperación.

- Se evita que personas con otras culturas organizacionales e ideas se incorporen a la organización.

\section{Fuentes Externas.}

Cuando no se consiguen suficientes candidatos calificados o las necesidades no se ajustan al 
perfil del puesto requerido en el ámbito interno, entonces las organizaciones se ven forzadas al reclutamiento externo mediante el internet y redes sociales.

\section{Aspectos positivos.}

- Atraer postulantes con conocimientos, experiencia laboral y formas nuevas de abordar los problemas internos de la organización.

- Aprovechar las inversiones en formación realizadas en otras empresas.

- Se puede dar el caso que resulta más económico contratar a un trabajador ya formado o calificado que invertir en uno de la misma organización.

\section{Aspectos negativos.}

- Duración excesiva del reclutamiento externo.

- Costo elevado del reclutamiento externo.

- Puede ser menos seguro.

- Puede originar frustración entre el personal de la organización que inspiraba ocupar la posición.

- Puede afectar a la política salarial de la empresa, produciendo desequilibrios remunerativos.

\section{Fuentes externas - métodos.}

\section{Presentación espontánea.}

La persona se convierte en candidato al acercarse a la Oficina de Personal de la organización. El proceso regular con estos postulantes es hacerles completar un formulario de solicitud de empleo con la información de su hoja de vida para alimentar la base de datos que se utilizará cuando se requiera cubrir una vacante. Tiene como ventaja el ser muy económico. No es recomendable utilizar este método para puestos gerenciales.

\section{Recomendaciones de los colaboradores.}

Tiene la ventaja que las personas ya vienen con cierta información sobre la organización y las características del puesto al que postulan. Además, los colaboradores recomiendan a personas con las que tienen cierta confianza que desempeñarán el trabajo eficientemente. Igualmente es un procedimiento menos costoso.

\section{La publicidad.}

Anunciar en los medios de comunicación social es una de las técnicas tradicionales más utilizadas para atraer candidatos por llegar a gran número de personas. Sin embargo, es más relacionado a cantidad que a calidad.

\section{Agencias de empleo especializadas.}

Uno de los medios más utilizados. Son organizaciones especializadas en reclutamiento y selección de personal. Pueden ser públicas o privadas. Su origen de las empresas consultoras puede ser nacional o internacional

\section{Organizaciones empresariales y colegios profesionales.}

Las empresas se dirigen a los colegios profesionales o a las asociaciones en las se agrupan las empresas de su sector de actividad con la finalidad que difundan la oferta de puestos. Las empresas se contactan con las organizaciones empresariales del país.

\section{Universidades y centros educativos.}

Los centros educativos y las Universidades son en muchos casos excelentes fuentes de reclutamiento. Se buscan a los estudiantes con mejores expedientes académicos y récord académico (quinto o tercio superior).

\section{Radio y televisión.}

No es un medio muy buscado debido a sus costos elevados, a pesar de dar una difusión masiva.

\section{Reclutamiento por internet y redes sociales.}

Cada vez más las empresas están acudiendo a las redes sociales como herramienta de reclutamiento y selección porque los anuncios son relativamente menos costosos, más dinámicos y a menudo, pueden dar resultados más rápido que otros medios.

\section{Selección de Personal Virtual}

\section{Conceptos de Selección de Personal}

- Para la Real Academia Española (RAE, s.f.) es "la acción y efecto de elegir a una 
o varias personas o cosas entre otras, separándolas de ellas y prefiriéndolas".

- Dolan et al. (2007) en su libro La Gestión de los Recursos Humanos indica que "es el proceso que comprende tanto la recopilación de información sobre los candidatos a un puesto de trabajo como la determinación de a quién deberá contratarse" (p. 129).

- Gómez-Mejía et al. (2008) en su libro Gestión de Recursos Humanos menciona que "es el proceso por el que se toma la decisión de 'contratar' a los candidatos a un puesto de trabajo es el segundo paso del proceso de contratación" (p. 190).

- El diagrama de flujo especifica las etapas del proceso de selección de personal, presencial o virtual.

\section{Personal}

Comité Evaluador de la Selección de

En las empresas se constituyen un Comité Evaluador conformado por los siguientes ejecutivos:

- Gerente General o su representante, quien preside.

- Gerente de Administración o Gerente de Finanzas.

- Gerente del Área interesada

- Jefe de la Asesoría Legal, y

- Jefe de Personal, quienes tienen la responsabilidad de conducir todo el proceso y demostrar inteligencia emocional y patrones de conducta de honestidad.

\section{Personal}

Convocatoria al proceso de Selección de

La Oficina de Personal realiza la convocatoria al proceso de selección de personal, mediante los medios utilizados en el reclutamiento de personal. Los interesados o postulantes toman conocimiento y deben adjuntar la información solicitada en la convocatoria y el perfil del puesto.

\section{Hoja de Vida, Curriculum Vitae y Oferta de Empleo}

La Hoja de Vida contiene fotografía del interesado, información breve del postulante, datos personales y sobre el perfil laboral o profesional, de preferencia en una hoja.

El Curriculum Vitae documentado contiene una información más detallada en la que se adjunta documentación sustentatoria de los estudios académicos, experiencia laboral, idiomas, certificados, constancias, datos personales y otros.

La Oferta de Empleo o Solicitud de Empleo es proporcionada por la empresa para ser llenado por el postulante, tiene la validez de una declaración jurada simple.

Esta etapa tiene carácter eliminatorio en caso de que no se cumplan los requisitos del puesto.

\section{Entrevista Preliminar virtual}

El Comité Evaluador después de haber verificado el cumplimiento de los requisitos mínimos exigidos para el puesto por el postulante, lo convoca en fecha y hora establecida mediante los canales virtuales en el correo electrónico para la entrevista preliminar.

La entrevista preliminar tiene un carácter exploratorio para ambas partes, para la empresa se evalúa el comportamiento del postulante con respecto a las siguientes habilidades blandas:

- Espíritu colaborativo

- Comunicación digital.

- Creatividad e innovación

- Eficacia y orden.

- Empatía y tolerancia.

- Resiliencia.

- Inteligencia Emocional.

- Trabajo en equipo

- Resolución de problemas

- Actitud positiva

- Honestidad.

- Capacidad de adaptarse al cambio.

- Tolerancia a la presión. 
Para el postulante es tomar información sobre la visión, misión y organización de la empresa, funciones del puesto, condiciones de trabajo, entre otra información.

Esta etapa tiene carácter eliminatorio.

\section{Prueba de Conocimientos virtual}

Esta prueba de conocimientos puede ser teórica y práctica según el puesto de trabajo.

Su elaboración es de responsabilidad de la Gerencia interesada en cubrir el puesto de trabajo, para garantizar su imparcialidad se recomienda la presentación de tres alternativas en sobre cerrado, si es teórica para que la Comisión decida. Es importante fijar el tiempo y el correo electrónico de su envió por él postulante. Esta etapa tiene carácter eliminatorio.

\section{Prueba Psicotécnica virtual}

Corresponde a los profesionales Psicólogos su elaboración e interpretación. Algunos tipos de pruebas psicológicas en un proceso de selección de personal.

\section{Test de Wartegg.}

Este test busca identificar la personalidad del postulante y su capacidad de resolver problemas de organización.

\section{Test de persona bajo la lluvia.}

Consiste en dibujar una persona bajo la lluvia, en una situación de estrés (la lluvia), el candidato recurre a defensas (paraguas), lo que permite observar y entender su personalidad.

\section{Test de la figura humana.}

El postulante en una hoja en blanco y un lápiz, le piden que dibuje una persona. Este test es proyectivo el psicólogo irá viendo los trazados de los dibujos las características de la personalidad.

\section{Test de series de dominó.}

Sirve para analizar la capacidad del postulante para elaborar conceptos y resolver los problemas.

\section{Test de Lüscher o de los colores.}

Busca conocer la personalidad del postulante a través de la elección de un color.
Las pruebas psicotécnicas por lo general no tienen puntaje asignado.

\section{Entrevista Final virtual por competencias}

Se puede realizar a través de plataformas tecnológicas virtuales como Google Meet, Zoom, Collaborate, etc. (sala pública participa la Comisión evaluadora y el entrevistado y la sala privada en la que deliberan los miembros de la Comisión). Las preguntas pueden ser estructuradas (se elaboran baterías de preguntas con anticipación) y no estructuradas (las preguntas fluyen de la entrevista).

\section{Objetivos.}

- Confirmar los datos de la hoja de vida y del CV sobre: datos personales, formación académica, experiencia laboral, conocimientos, rasgos de personalidad y carácter, etc.

- Conocer los proyectos y pretensiones económicas del candidato.

- Conocer los valores, capacidades y habilidades blandas del candidato y su actitud frente al proceso de reclutamiento y selección de personal.

- Saber si se adapta a las necesidades de la empresa y a los requerimientos del puesto.

- Obtener la máxima cantidad de información para saber la adaptabilidad del entrevistado a la posición que se oferta.

Esta etapa tiene carácter eliminatorio.

\section{Contratación}

El postulante que obtenga la mayor calificación en el proceso de selección de personal es declarado ganador, si por algún inconveniente no pueda sumir el puesto, se designara al siguiente en orden de prelación.

La Oficina de Asesoría Legal de la Empresa formalizara el contrato de trabajo de acuerdo con la legislación laboral vigente.

\section{Pasantía}

En esta última etapa el colaborador contratado se apersona a la Oficina de Personal para: 
- Firmar su contrato de trabajo.

- Recibir el Reglamento Interno de Trabajo de la Empresa.

- Recibir el documento de presentación oficial como colaborador

- De acuerdo con la política laboral de la empresa puede hacer una pasantía por las diversas áreas u órganos de la entidad y/o recibir conferencias sobre la historia y cultura organizacional de la empresa.

Tabla de Calificación del Proceso de Selección de Personal

El Comité Evaluador del proceso de selección de personal evalúa y califica a los postulantes en cada una de las etapas del proceso técnico descrito, se elaboran cuatro tablas de calificación para cada grupo ocupacional: directivos, profesionales, técnicos y administrativos - servicios. Ejemplo:

\section{Grupo Ocupacional Directivo (Puntaje Máxi- mo 100 puntos)}

I. Evaluación del Curriculum Vitae: (puntaje máximo 30), los puntajes no son acumulativos.

\section{Formación académica:}

Grados académicos, títulos profesionales y diplomados (puntaje máximo 15)

- Grado de Doctor (6 puntos)

- Grado de Maestro, precisando la Mención (5 puntos)

- Segunda Especialidad (4 puntos)

- Título Profesional (4 puntos)

- Grado Académico Bachiller (2 puntos)

- Diplomados otorgados por Universidades $\mathrm{c} / \mathrm{u}(1$ punto)

- Semestres Académicos de Postgrado c/u (1 punto)

Los grados, títulos y especialización deben estar registrados en la SUNEDU y los otorgados por Universidades extranjeras deben ser revalidados por una Universidad autorizada y registrada en el Ministerio de Educación.

2. Experiencia laboral (puntaje máximo 10)

- Experiencia profesional en puestos directivos (1 punto por año)

- Experiencia laboral en la actividad privada ( 0.50 puntos por año)

Se acreditarán con certificados de trabajo y los puntajes no son acumulativos.

\section{Idiomas (puntaje máximo 5).}

Se acredita con constancia o certificado. Los puntajes no son acumulativos.

- A nivel básico (1 punto)

- A nivel intermedio (3 puntos)

- A nivel avanzado (5 puntos)

II. Entrevista Personal: se realizan con preguntas estructuradas o no estructuradas, abiertas o cerradas. (puntaje máximo 40), como por ejemplo:

- Cómo te defines como persona.

- Por qué has escogido para postular a esta Empresa.

- Cuáles son tus fortalezas y debilidades.

- Consideras que eres la persona adecuada para el puesto.

- Cuáles son los motivos por la que dejastes o dejas tu trabajo.

- Cómo manejas los conflictos laborales.

- Dónde y cómo te ves en cinco años.

- Tienes conocimientos del puesto de trabajo.

- Usas programas y equipos informáticos.

- Habla, lee y escribe en un idioma extranjero.

- Cuáles son tus experiencias laborales anteriores.

- Cuál es tu formación profesional. 
- También se puede aplicar una entrevista psicológica

\section{Escala de evaluación:}

- Excelente. (40 puntos)

- Muy Buena. (30 puntos)

- Regular. (20 puntos)

- Mala. (0 puntos)

III. Pruebas de conocimiento por competencia (puntaje máximo 30).

Evaluación de Conocimientos Teóricos o Pruebas Técnicas o

- Dinámica de grupos

- Desarrollo de casos prácticos

- Evaluación de uso de programas y equipos informáticos: (Programas Informáticos: Microsoft office, bases de datos. Equipos Ordenadores fijos y portátiles, Tablet, Smartphone).

El Comité evaluador definirá el tipo de prueba o de pruebas según el puesto (ver Tabla 1).

Tabla 1.

Resumen de la calificación

\begin{tabular}{ll}
\hline Evaluación Curriculum Vitae & 30 puntos \\
Entrevista Personal & 40 puntos \\
Pruebas de Conocimiento & 30 puntos \\
\hline Total & 100 puntos \\
\hline
\end{tabular}

Firmas del Comité Evaluador

Fecha de la evaluación

\section{CONCLUSIONES}

Las empresas tienen su agenda de desarrollo reflejada en sus planes estratégicos y tácticos para incorporar nuevos colaboradores.

Las empresas para lograr liderazgo y competitividad tienen que apostar por el cambio y requieren contratar personal con creatividad e innovación.

Las empresas para lograr la transformación digital y construir sus propias plataformas tecnológicas deben atraer personal calificado y bien remunerado.

La meritocracia aplicada al ámbito empresarial es la única forma de incorporar personal con las competencias laborales que garanticen una eficiente, eficaz y efectiva selección de personal.

\section{REFERENCIAS BIBLIOGRÁFICAS}

Dolan, S. L., Valle Cabrera, R., Jackson, S. E. y Schuler, R. S. (2007). Gestión de Recursos Humanos: Cómo atraer, retener y desarrollar con éxito el capital humano en tiempos de transformación (3a ed.). Editorial Mac Graw Hill.

Gómez-Mejía, L. R., Balkin, D. B. y Cardy, R. L. (2008). Gestión de Recursos Humanos (5a ed.). Pearson Prentice Hall.

Real Academia Española. (s.f.). Reclutamiento. En Diccionario de la lengua española. Recuperado en 10 de julio de 2021, de https://dle.rae.es/reclutamiento

Real Academia Española. (s.f.). Selección. En Diccionario de la lengua española. Recuperado en 10 de julio de 2021, de https://dle.rae.es/selección 